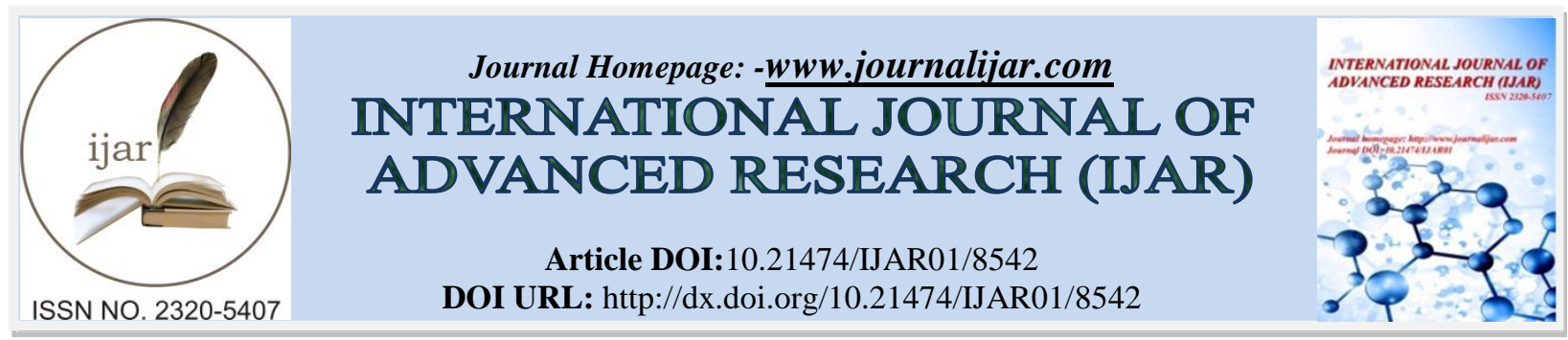

RESEARCH ARTICLE

\title{
CONTRIBUTION OF AUTONOMIC NERVOUS SYSTEM EXPLORATION IN THE DIAGNOSIS OF SYMPTOMATIC SINUS BRADYCARDIA-A REPORT OF TWO CASES.
}

\author{
Lalla Fatima Ezzahra El-Hassani ${ }^{1}$, Amine Ahmindach ${ }^{1}$, Ilyas El-Kassimi ${ }^{2}$, Salwa Cheraou ${ }^{1}$, Halima \\ Benjelloun $^{3}$, Roukia Fellat ${ }^{3}$ and Mohamed Cherti ${ }^{1}$. \\ 1. Department of Cardiology "B", Ibn Sina University Hospital Center, Rabat, Morocco. \\ 2. Department of Internal Medicine, Mohammed V Military teaching Hospital, Rabat, Morocco. \\ 3. Department of Cardiology "A”, Ibn Sina University Hospital Center, Rabat, Morocco.
}

\section{Manuscript Info}

Manuscript History

Received: 11 December 2018

Final Accepted: 13 January 2019

Published: February 2019

Key words:-

Autonomic nervous system; Sinus

bradycardia; Electrophysiological

exploration.

\section{Abstract}

A symptomatic sinus bradycardia is a very common situation in cardiology for which the diagnostic and therapeutic management are far from being well codified. After ruling out the obvious and reversible causes, the differential diagnosis between sinus bradycardia caused by vagal hypertonia and sinus dysfunction is often not easy subjecting the patient to electrophysiological exploration. We report 2 cases of permanent sinus bradycardia for which a non-invasive exploration of the autonomic nervous system (ANS) has led to the diagnosis of vagal hypertone bradycardia, in the first case after electrophysiological exploration, whereas in the second case the electrophysiological study was not performed.

Copy Right, IJAR, 2019,. All rights reserved.

\section{Introduction:-}

Symptomatic sinus bradycardia (SB) is a special entity whose prognosis depends strongly on etiology. Even after ruling out the obvious and reversible causes, in its etiological diagnosis approach, it is often difficult to differentiate a SB caused by benign vagal hypertonia from an organic sinus dysfunction requiring the implantation of a Pace maker. In order to cope with this dilemma, it is often necessary to resort to invasive complementary explorations which may in some cases fail to reveal a specific etiology; while a simple detailed study of the autonomic nervous system (ANS) could contribute to the diagnosis and put the patient on the right therapeutic path.

\section{Case Reports}

Case 1

A 51-year-old woman started complaining of extreme fatigue associated with migraine six months before her visit to our department for which she was prescribed vitamins and paracetamol by her family physician. She has no medical history and did not take any bradycardic medication. She denies chest pain, breathlessness or syncope. The physical examination was normal except for a regular heart rate (HR) of 40 beat per minute (bpm). Her blood pressure was $110 / 60 \mathrm{mmHg}$, with a heart rate of $40 \mathrm{bpm}$. Cardiovascular and pulmonary auscultation were normal, thyroid palpation showed no node or thyroid enlargement, abdominal and neurological examinations were normal Her ECG showed a sinus bradycardia of $45 \mathrm{bpm}$, heart axis at $-19^{\circ}$, PR of $140 \mathrm{~ms}$, thin QRS and normal QTc (Figure 1).

Chest x-ray was normal, blood tests including troponin, renal function, hepatic function, TSH, electrolytes, calcium and phosphore were normal. Echocardiography showed a normal heart structure and function. Holter ECG showed a 
sinus bradycardia during most of the nycthemer (daytime average HR of $47 \mathrm{bpm}$ and nocturnal average HR of 35 bpm) interspersed by few episodes of sinus tachycardia with a maximum HR of $130 \mathrm{bpm}$, it showed no atrial or ventricular arrythmias nor conduction abnormalities, the QT was normal. Exercise testing showed an increase in HR from $45 \mathrm{bpm}$ at rest to $110 \mathrm{bpm}$ at the first level, the test was stopped at the third level of Bruce protocol at $85 \%$ of the theorical maximal heart rate for shortness of breath with no ischemic or rhythmic events.

The electrophysiological study (EP study) in Figures 2 to 5 does not find any evidence for an intrinsic impairment of sinus function or atrioventricular conduction, namely: the conduction intervals in the basic state are normal with $\mathrm{AH}=116 \mathrm{~ms}, \mathrm{HV}=55 \mathrm{~ms}$, the corrected sinus node recovery time is normal (CSRT) at $134 \mathrm{~ms}$, eliminating sinus dysfunction. The anterograde refractory period of the atrioventricular node or Wenckebach point is $680 \mathrm{~ms}$ with a suprahissian block. The administration of atropine leads to an acceleration of heart rate from 47 to $73 \mathrm{bpm}$. The carotid massage on the right and the left does not trigger any abnormality.

Exploration of the autonomous nervous system (ANS) was requested to establish a diagnosis, and showed the following autonomic profile: Vagal hyperactivity, central and peripheral alpha sympathetic hyperactivity, weak central and peripheral beta sympathetic activity and a mild baroreceptor abnormality. The patient was prescribed hygienic and dietary measures including high liquid intake, salt-enriched diet, compression stockings and pharmacological therapy phenobarbital $10 \mathrm{mg}$ bid which allowed relieving the symptoms and raising the HR to 55 bpm. The follow-up at 9 months is satisfactory.

\section{Case 2}

A 48-year-old former smoker male was referred for symptomatic bradycardia. He complained of headaches followed in neurology for 7 years. His headaches are associated with sweating, vomiting and vertigo that worsen in times of

\section{Open Acess}

creativecommons.org/licenses/by-nc/4.0/), which permits reuse, distribution and reproduction of the article, provided that the original work is properly cited and the reuse is restricted to noncommercial purposes. For commercial reuse, contact reprints@pulsus.com

Clin Cardiol J Vol 3 No 1 January 2019

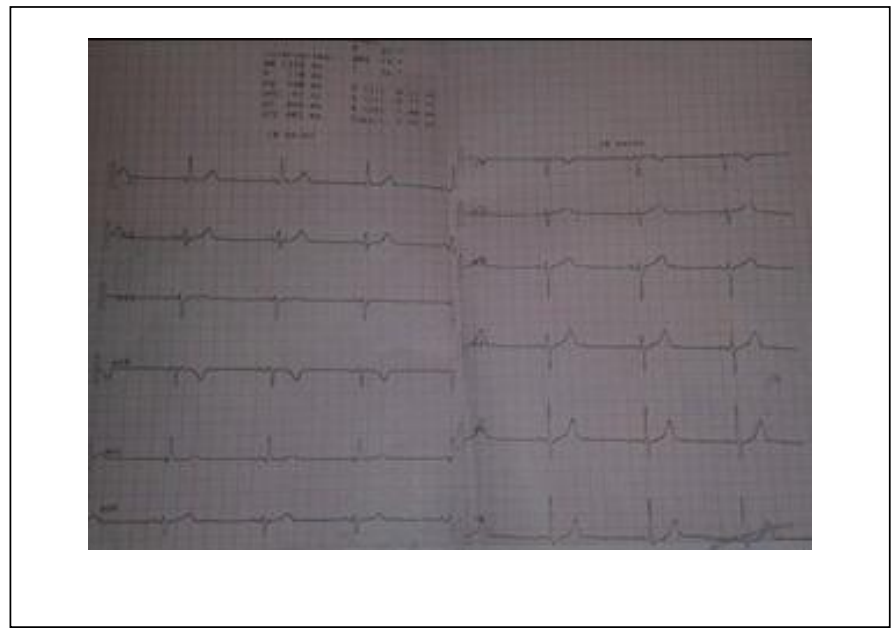

Figure 1:-12 leads ECG showing an isolated sinus bradycardia 


\section{El-Hassani LFE et al}
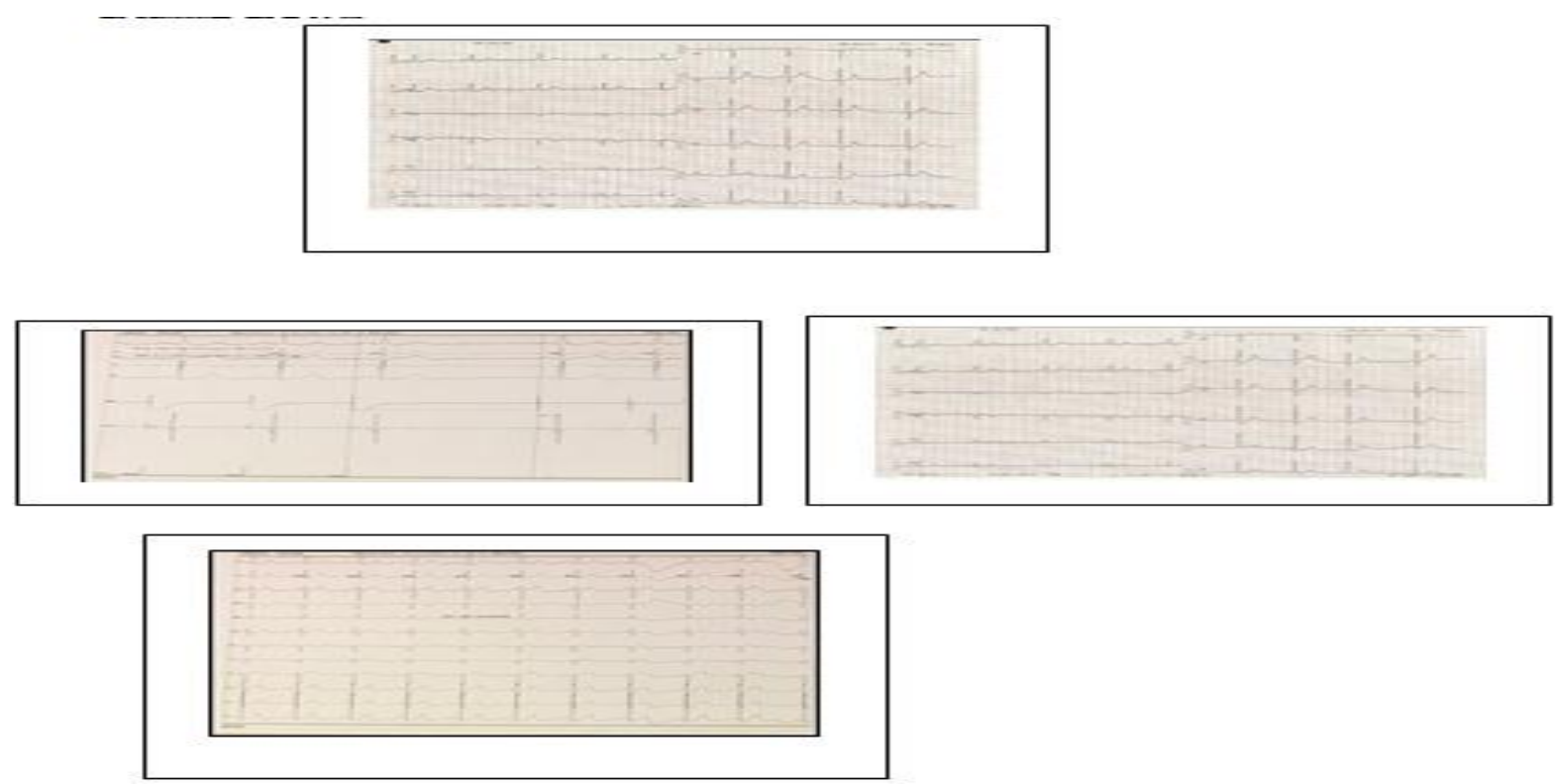

stress and after insomnia. The presence of familial bradycardia (sister and son) was noted. Physical examination was normal, the ECG showed a sinus bradycardia at $48 \mathrm{bpm}$, cardiac ultrasound imaging was normal, Holter ECG showed daytime sinus bradycardia as low as $34 \mathrm{bpm}$ associated with pauses of less than 2 seconds. Exercise test showed a good adaptation of heart rate and blood pressure to stress. The ANS exploration found an increased vagal tone associated to a central $\alpha$ and $\beta$ sympathetic hyperactivity. A phenobarbital- based treatment was administered with good clinical evolution (Figure 6).

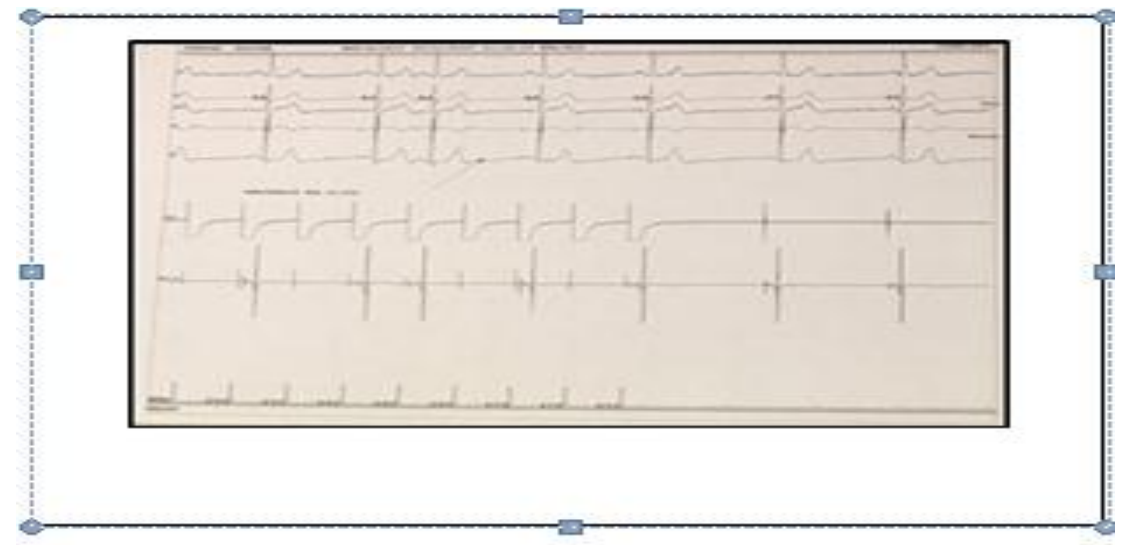

\section{Discussion:-}

Sinus bradycardia (SB) is defined as an electrical impulse that originates in the sinus node but with a frequency of less than 60 cycles per minute. It is mild between 50 and $59 \mathrm{bpm}$, moderate between 40 and $49 \mathrm{bpm}$ and severe below $40 \mathrm{bpm}$. It can be caused by either an organicsinus node involvement (degenerative, ischemic, infiltrative, and inflammatory), or a functional impairment: (drug intake, dyskaliemia, hypothyroidism, hypothermia, hypoglycemia, peptic ulcer or vagal hypertonia).

The sinus node ( $\mathrm{SN}$ ) is the heart's main pacemaker, its cells have the ability to automatically generate a spontaneous action potential at a frequency of 80 to 100 cycles per minute by means of the pacemaker current (If) responsible for a 
slow and spontaneous diastolic depolarization until reaching the activation threshold of an action potential that propagates in the rest of the cardiac conductive tissue. To adapt this intrinsic automatism of the heart to different physiological states, $\mathrm{SN}$ is regulated by the autonomous nervous system (ANS). The parasympathetic system permanently slows down the sinus node through the right vagus nerve acting through acetylcholine by slowing down the spontaneous diastolic depolarization slope resulting in a decrease in sinus node impulse frequency and therefore heart rate (HR). In contrast, the sympathetic system, which has as the main neurotransmitter norepinephrine, acts on the beta-adrenergic receptors of SN and accelerates the spontaneous diastolic depolarization slope, thus increasing the SN impulse frequency and the HR.

SB is not always pathological, it can be observed in physiological states as in the elderly and endurance athletes. Although pathological, it can remain, often, asymptomatic and is found only accidentally; its tolerance is variable clinically, a slow heart rate remains mostly of no consequence when it is moderate or settles progressively, if bradycardia is extreme or occurs suddenly, the clinical scene may be unambiguous. In this case, nonspecific and variable symptoms may appear: brief discomfort with lightheadedness, dizziness, presyncope and syncope. When an underlying cardiac disease ispresent, SB can manifest as angina or congestive heart failure. Finally, in the paucisymptomatic forms, the symptoms can be translated as asthenia, intolerance to the effort or an ideomotor slowing down (1). The SB represents a particular entity whose diagnosis can be suspected on clinical examination and confirmed with the ECG or the ECG Holter if intermittent.

The etiological assessment for a reversible cause includes a good history, a complete clinical examination, an ECG, a biological assessment (electrolytes, magnesium, calcium, glycose, thyroid status and troponin) and transthoracic echocardiography if structural heart disease is suspected.

In case of normal initial assessment and in the absence of an obvious cause for bradycardia, the differential diagnosis between sinus dysfunction and sinus bradycardia due to autonomic dysfonction gain a foothold. Other $2^{\text {nd }}$ line investigations seek to confirm or refute the organic involvement of the SNsùimposer. The ECG Holter is the test of choice for the diagnosis of sinus dysfunction with a $100 \%$ specificity when it demonstrates sinus pause/stop, sinoatrial block or alternating bradycardia-tachycardia. But it is not uncommon for the Holter ECG to remain inconclusive by finding only sinus bradycardia that can be permanent or intermittent. The stress test may be useful in the case of symptoms of stress in search of chronotropic insufficiency, but its diagnostic value is low for the diagnosis of other types of arrhythmias in sinus dysfunction $(2,3)$.

The pharmacological test for the study of intrinsic sinus function after blocking autonomic nervous systeminfluences by intravenous use of atropine and propranolol (4) has low sensitivity and specificity for the diagnosis of sinus dysfunction due to a large individual variability in the response to this test. The place of this test is very limited in case of intermittent sinus bradycardia.

Electrophysiological study explores sinus function mainly by measuring the corrected sinus node recovery time (CSRT) by the Mandel method (5). According to the 2018 syncope ESC recommendations, a CSRT greater than 525 ms supports organ dysfunction of the sinus node (6). The prognostic value of this method is poorly defined, especially since CSRT may be lengthened in case of vagal hypertonia (7). Several studies have shown that dysautonomia associated with organic involvement of the sinus node plays an important role in the onset of symptoms (8-10). Electrophysiological Investigation will be reserved for patients with SB who have experienced syncope under specific conditions (supine, exercise, or palpitations), patient with underlying heart disease, and patients with bifascicular block.

In the first case we report, electrophysiological study was in favor of sinus bradycardia by vagal hypertonia as it showed a 134 msCSRT and an elevation of the heart rate from 47 to $73 \mathrm{bpm}$ after blockade of autonomic control by atropine. To confirm the involvement of the autonomic nervous system, we used an exploration of this system which confirmed the vagal hypertonia. However, in the second case, we preferred to perform a cardiovascular exploration of the autonomous nervous system before exploring the sinus node invasively which allowed us to make the diagnosis of a sinus bradycardia by vagal hypertonia with great certainty and exempt the patient from an invasive gesture.

Cardiovascular exploration of autonomous nervous system is based on the study of variability in heart rate and blood pressure during various ANS stimulation tests. Tests such as Hand grip, mental stress and orthostatic testing explore 
the sympathetic system while the variability of heart rate during deep breathing, Valsalva and the initial phase of the orthostatic test provide information on the functioning of the parasympathetic system.

A broad consensus places the deep breathing test as the pillar test for the exploration of parasympathetic function (11-14). This test explores the variability of heart rate (HR) over 6 cycles of inspiration and deep expiration lasting 5 seconds each. During inspiration the vagal tone is lifted, and the heart rate rises causing a sinus arrhythmia. Measuring this variability allows a quantitative approach to vagal activity. The ratio $\mathrm{HR}$ at expiration/HR at inspiration of $30 \%$ is considered normal; variability above $30 \%$ refers to a vagal hypertone.

\section{Conclusion:-}

In the two present cases, the diagnosis of vagal hypertonia was made in the deep breathing test which showed an exaggerated sinus arrhythmia and in the orthostatic test which showed a significant increase in cardiac HR exceeding $20 \%$ by lifting the vagal brake during the first few seconds after standing up.

\section{References:-}

1. Abbott JA, Hirschfeld DS, Kunkel FW, et al. Graded exercise testing in patients with sinus node dysfunction. The Amer J Med. 1977;62:330-338.

2. Johnston FA, Robinson JF, Fyfe T, et al. Exercise testing in the diagnosis of sick sinus syndrome in the elderly: implications for treatment. Pacing Clin Electrophy. 1987;10:831-838.

3. Bruce L, Wilkoff MD, Raymond E, et al. Exercise testing for chronotropic assessment. Cardiology Clinics. 1992;705-717.

4. Jose AD, Taylor RR. Autonomic blockade by propranolol and atropine to study intrinsic myocardial function in man. J Clin Invest. 1969;48:2019-2031.

5. Mandel WJ, Hayakava H, Allen HN, et al. Assessment of sinus node function in patients with the sick sinus syndrome. Circulation. 1972;46:761-769.

6. 2018 ESC Guidelines for the diagnosis and management of syncope. Eur Heart J. 2018;1-69.

7. Graff B, Graff G, Koźluk E, et al. Electrophysiological features in patients with sinus node dysfunction and vasovagal syncope. Arch Med Sci. 2011;6: 963-970.

8. Brignole M, Menozzi C, Gianfranchi L, et al. Neurally mediated syncope detected by carotid sinus massage and head-up tilt test in sick sinus syndrome. Am J Cardiol. 1991;68:1032-1036.

9. Alboni P, Menozzi C, Brignole M, et al. An abnormal neural reflex plays a role in causing syncope in sinus bradycardia. J Am Coll Cardiol. 1993;22:1130-1134.

10. Pachon JC, Pachon EI, Pachon JC, et al. Cardioneuroablation new treatment for neurocardiogenic syncope, functional AV block and sinus dysfunction using catheter RF-ablation. Europace. 2005;7(1):1-13.

11. Baschieri F, Calandra-Buonaura G, Doria A, et al. Cardiovascular autonomic testing performed with a new integrated instrumental approach is useful in differentiating MSA-P from PD at an early stage. Parkinsonism Relat Disord. 2015;21:477-482.

12. Rocchi C, Pierantozzi M, Galati S, et al. Autonomic function tests and MIBG in Parkinson's disease: correlation to disease duration and motor symptoms. CNS Neurosci Ther. 2015;21:727-732.

13. Aboudrar S, Benjelloun H, Benazzouz A, et al. Evaluation of the vagal activity by the Deep-Breathing test. Neurophysiol Clin. 2007;37(1):41-46.

14. Souad A, Hanane R, Leila E, et al. Young footballers, assessed by deep breathing test, has a higher vagal response. Open J of Mol \& Intg Phy. 2012;2:61-64. 